\title{
Sustaining the Life of Wireless Sensor Node with Energy Harvesting
}

\author{
S. Viswanatha Rao, Sakuntala S. Pillai, Shiny G.
}

\begin{abstract}
Wireless Sensor Networks (WSN) play a significant role in a number of sensing and monitoring applications. It has also become a key enabling technology for Internet of Things (IoT). Most of the earlier works were focused on extending the battery life by reducing the energy consumption of the wireless node. Availability of small footprint devices capable of ambient energy harvesting has triggered renewed interest in enhancing the performance of WSNs. In this paper we propose a novel method that can be applied to duty cycle based Media Access Control (MAC) protocols in energy harvesting WSNs to sustain the life of the node. Our approach takes into consideration the solar energy available at any instant of time so as to maximize the performance. We have conducted detailed measurements and analysis of solar energy harvested under different conditions. Our results show significant enhancement in throughput achieved while sustaining the life of the node.
\end{abstract}

Keywords: duty cycle, media access control, solar energy harvesting, wireless sensor networks.

\section{INTRODUCTION}

$\mathrm{W}_{\mathrm{i}}$ ireless Sensor Networks (WSNs) are formed by large number of nodes capable of communicating with each other wirelessly. Conventionally, the wireless nodes are activated using batteries. Limited energy capacity of the battery is a constraint on the life of the node. It is not practical to replace the batteries due to the hostile application environment and the large number of nodes being deployed. Hence, the energy consumption by the nodes has to be kept at a bare minimum in order to have a prolonged life for these networks. A number of techniques were developed towards achieving this objective in terms of physical design as well as optimisation at different layers of software.

Conventional WSNs have been researched extensively in literature [1], [2]. MAC layer has been one of the more specific areas that have been addressed by researchers in order to reduce energy consumption in Wireless Sensor Nodes [3]. Among the different protocols addressed in MAC, controlling power consumption by different duty cycling schemes has been very popularly dealt with.

With the key focus on reducing the power consumption,

Revised Manuscript Received on December 05, 2019

* Correspondence Author

S. Viswanatha Rao*, Dept. of ECE, Mar Baselios College of Engineering \& Technology, Thiruvananthapuram, India. Email: rao-sv@ hotmail.com

Sakuntala S. Pillai, Former Dean(R\&D), Mar Baselios College of Engineering \& Technology, Thiruvananthapuram, India. Email: sakuntala.pillai@gmail.com

Shiny G., Dept. of ECE, College of Engineering \& Technology, Thiruvananthapuram, India. Email: drgshiny@gmail.com performance in terms of throughput and latency are compromised in many cases. Recent studies show that ambient sources such as solar, vibration and thermal can be made use of for energising the wireless nodes. Though these ambient energy sources are unpredictable and inconsistent in nature, they can be utilised by properly converting and storing the energy or by supplementing the conventional sources such as the battery.

In this paper we analyse how the solar energy can be effectively utilised to sustain the life of WSNs. In addition, we demonstrate using Sensor-MAC (SMAC), a widely used MAC protocol, that there is significant enhancement in throughput of the network.

The rest of the paper is organised as follows. Prior art related to energy harvesting WSNs is discussed in the next section. Section III details our solar energy measurements and subsequent analysis. A solar panel suitable for a WSN node was used for the measurements. Section IV analyses the performance of SMAC to find out parameters such as data rate, duty cycle and energy consumed corresponding to a desired throughput. The energy model and its application to SMAC to arrive at extended life of the wireless node is detailed in Section V. Results are discussed in section VI. Finally, the paper concludes with section VII.

\section{RELATED WORK}

There has been continued research work related to solar energy harvesting for WSNs and studies related to performance of MAC. In [4], the authors have analysed the potential of solar cell to provide energy to wireless node. A solar tracking mechanism to improve the energy harvested is presented in [5]. Different Maximum Power Tracking (MPPT) techniques are explored in [6] with focus on Semi-Pilot Cell Fractional Open Circuit Voltage (SPC-FOCV). As mentioned above, there are independent works related to solar harvesting, its enhancement, and corresponding generic duty cycle approaches to MAC for WSNs. O. Yang and W.B. Heinzelman [7] have developed a model to analyse the performance of duty-cycled MAC protocols that includes SMAC. In [8], the authors have proposed a queuing based model for solar energy to analyse the performance of SMAC. Most of the works available in literature are either intended to minimize energy consumption or models to analyse the performance. Whereas our work is based on measured values of solar energy and is focused on effective usage of harvested energy to increase performance while extending the life of the wireless node. 


\section{Sustaining the Life of Wireless Sensor Node with Energy Harvesting}

In our work, we have developed an approach that helps to increase the throughput of duty cycle based MAC protocols under solar energy harvesting environment.

\section{SOLAR ENERGY MEASUREMENTS AND ANALYSIS}

Solar has become one of the most significant sources of ambient energy. It should be noted that there is always a variation in the intensity of solar radiation depending on the geographical location, in addition to the temporal variations. We conducted measurements to find out the amount of energy that could be harvested from mini solar panels that could be used for activating wireless sensors. Solar power was measured at 30 minutes intervals to cover a total duration of 24 hours representing one day. A mini solar panel having dimensions of $60 \mathrm{~mm} \times 50 \mathrm{~mm}$ was used for carrying out the measurements. Observations were made under different scenarios. This includes measurements during sunny days, cloudy days and under passing clouds to capture steady as well as fluctuations in energy harvested.

The solar power measured at regular intervals of 30 minutes for the day is shown in fig. 1. The load was set to 42 ohms to maximize the power. This load was identified by making measurements across a wide range of resistance values. It can be seen that the solar power available reduces drastically in the presence of clouds.

We have used these measurements to analyse how effectively the harvested solar energy can be utilized to enhance throughput, in addition to extending the life of the wireless node. We have selected two representative conditions for rest of our analysis. The first one is a sunny day with maximum solar radiation that we denote as Scenario EH1 and the second one is a cloudy day with medium sunlight denoted as Scenario EH2. In addition, we have a third condition during night time, which is implicit, where there is no energy harvested. We have used SMAC as the MAC protocol for our study as it is one of the popularly used MAC for many WSN applications.

\section{PERFORMANCE OF SMAC}

SMAC is a synchronous MAC protocol that is widely implemented in WSNs[9]. In the following sections we

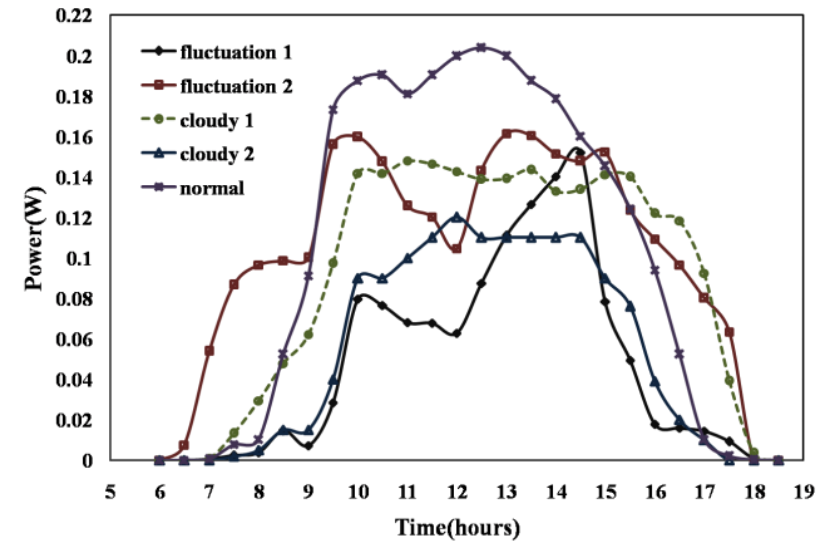

Fig. 1. Solar power measurements over a day, under different weather conditions.

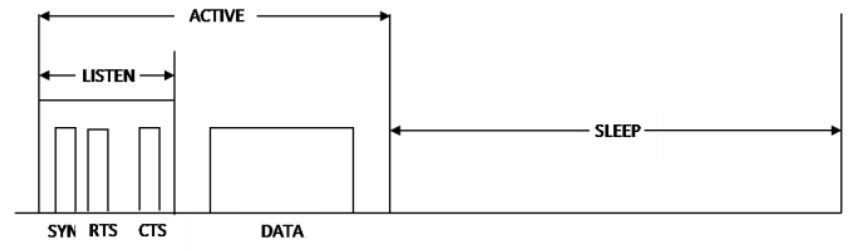

Fig. 2. SMAC frame showing active and sleep periods

analyse using SMAC, the impact of data rate and duty cycle on throughput of a wireless node from an energy harvesting perspective.

\section{A. SMAC Protocol}

SMAC is specifically designed to take care of the requirements of Wireless Sensor Networks. A frame in SMAC is made up of an active period and a sleep period as shown in fig 2.

The handshaking, data transmission and reception take place within the active period. Thus substantial amount of energy is consumed during this period whereas minimum energy is required in sleep period to keep the wireless node alive. In our work, the consumption of energy is controlled by varying the active period of the SMAC depending on the quantity of energy available through harvesting.

\section{B. Simulation Set-up}

Simulations were conducted in ns 2 with a star topology to find out the energy consumed by a wireless node. The energy available in a node was obtained considering the measured values of solar energy harvested. SMAC was used for the MAC protocol. A transmit power of $0.1414 \mathrm{~W}$, receive power of $0.1012 \mathrm{~W}$, idle power of $1.8 \mathrm{~mW}$ and sleep power of $18 \mathrm{uW}$ were used taking into consideration the values of typical commercial devices available. Other simulation parameters are given in Table I.

\section{Impact of Data Rate and Energy Consumed on Throughput}

We have conducted studies to find out the throughput of SMAC under varying data rates and against different duty cycles. The throughput increases as the data rate increases until a cut-off point after which it remains the same. We have noticed that at lower duty cycles the throughput reaches a saturation point earlier compared to higher duty cycles. Thus, though the throughput can be increased by increasing both duty cycle as well as data rate, there is a cut-off data rate beyond which the throughput cannot be increased.

In order to assess the energy consumed, we have conducted simulations at different data rates and duty cycles. Energy consumed increases as the duty cycle is increased. This is as expected because more energy is consumed when the active period is increased. The energy consumed reaches a steady value in spite of increasing the data rates. This is due to the fact that the active period available for a given duty cycle limits the maximum number of transmissions and receptions possible. 
The studies conducted on throughput and energy consumption for a wireless node for different data rates as well as duty cycles provide us an insight into the required data rate,

TABLE I

SYSTEM PARAMETERS

\begin{tabular}{|l|l|}
\hline Topology area & $500 \mathrm{~m} \times 500 \mathrm{~m}$ \\
\hline Propagation Model & Two ray ground model \\
\hline Bandwidth & $11 \mathrm{MHz}$ \\
\hline Frequency & $2.4 \mathrm{GHz}$ \\
\hline Routing Protocol & AODV \\
\hline Queue length & 50 \\
\hline Initial Energy & $2500 \mathrm{~J}$ \\
\hline Traffic & $\mathrm{CBR}$ \\
\hline Duty Cycle & $20 \%-90 \%$ \\
\hline Simulation time (single run) & $1800 \mathrm{~s}$ \\
\hline
\end{tabular}

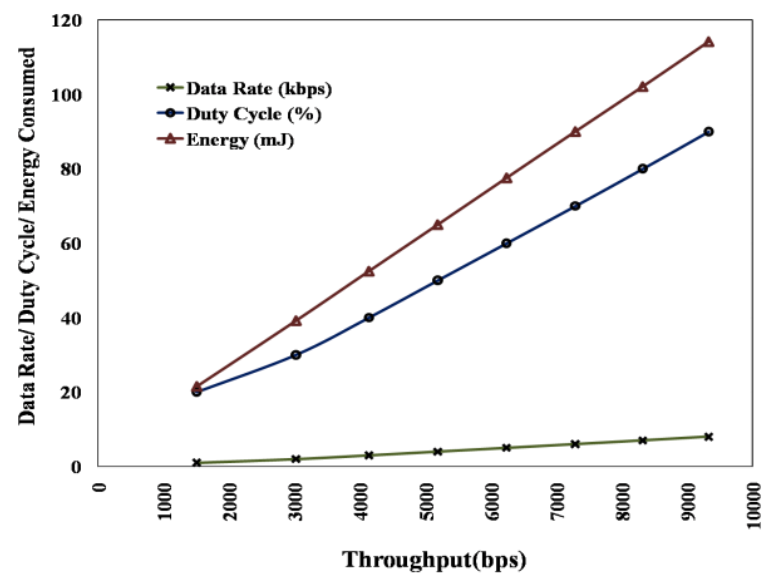

Fig. 3. Energy, duty cycle and data rate required for desired throughput

duty cycle and the corresponding energy required so as to obtain a specific throughput. This is depicted in fig. 3 . Alternatively, we can also find out for a given energy that is available, the corresponding data rate and duty cycle that will provide the maximum throughput. Fig. 4 illustrates the cumulative energy consumed and that harvested over a period of 24 hours. The cumulative energy that is utilised by the node during the operation at various duty cycles increases linearly, but with different slopes depending on the amount of

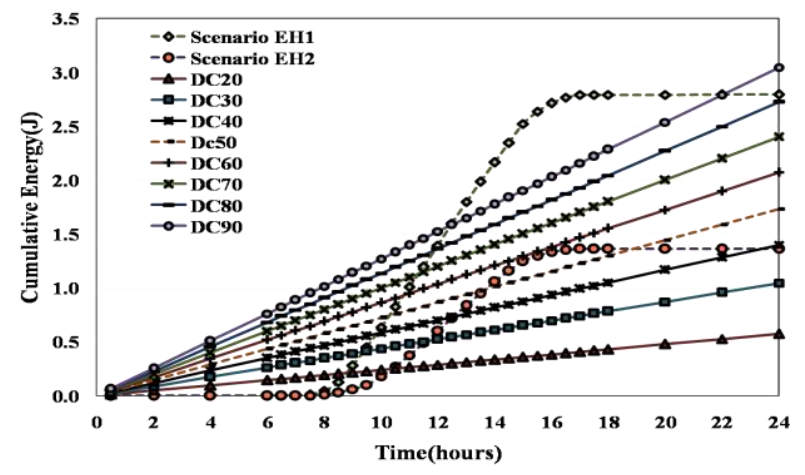

Fig. 4. Cumulative energy consumed and harvested over 24 hours energy consumed. The cumulative energy harvested has a zero slope initially as there is no energy harvested in the absence of sunlight during night hours. The energy builds up subsequently with the availability of solar radiance and again flattens towards the end of the daytime. It can be seen that the total energy harvested over one day is more than that consumed at lower duty cycles but not sufficient to operate the node at higher duty cycles for the entire day. Again, this depends on the weather conditions prevailing during the day. It can be seen that when operated using harvested energy, the wireless node is required to operate under different duty cycles to make efficient use of the energy that is harvested, as the energy available is not steady in nature. Hence, based on the energy harvested, it is important to adapt the duty cycle to prolong the life of the wireless node.

\section{THE ENERGY MODEL}

We have looked at the energy model of a wireless node from three aspects, energy harvesting, energy consumption and energy discharge. The energy harvesting depends on the nature of ambient source. The energy harvested $\left(\mathrm{EH}_{\mathrm{t}}\right)$ at time $\mathrm{t}$ varies from a minimum of zero to a maximum value depending on the harvesting module and the solar radiation. We have assumed that the node is capable of storing the harvested energy. Again, the maximum energy that can be stored is limited by the battery capacity. We have considered a typical 3 Volts rechargeable coin cell with $250 \mathrm{mAh}$ capacity that could provide more than $2500 \mathrm{~J}$.

We define the term Energy Available $\left(\mathrm{EA}_{\mathrm{t}}\right)$ at any time $\mathrm{t}$ as the energy that is available in the chargeable battery at that instant of time $t$. The energy consumed by the node $\left(\mathrm{EC}_{\mathrm{t}}\right)$ at time $t$ depends on the active period that is related to the duty cycle at that time. $\mathrm{EA}_{\mathrm{t}}$ depends on $\mathrm{EH}_{\mathrm{t}}$ and the energy consumed $\left(\mathrm{EC}_{\mathrm{t}-1}\right)$ during the previous instant of time $(\mathrm{t}-1)$.

$\mathrm{EA}_{\mathrm{t}}=\mathrm{EH}_{\mathrm{t}}+\left(\mathrm{EA}_{\mathrm{t}-1}-\mathrm{EC}_{\mathrm{t}-1}\right)$

In order to do our performance analysis, we have divided the energy available in the node into different energy bands. Based on our simulation, the minimum energy required for a node to be operational with SMAC is $0.012 \mathrm{~J}$. For any value of $\mathrm{EA}_{\mathrm{t}}$ below this, the node will be inactive. We have kept the simulation period as 30 minutes. We vary the duty cycle from a minimum of $20 \%$ to a maximum of $90 \%$. We have found that energy of $1100 \mathrm{~J}$ is required to keep the node active at $20 \%$ duty cycle for a period of 24 hours without any harvested energy. This is a safe limit when compared to the duration of night period where no energy is available for harvesting. With this as a reference and $2500 \mathrm{~J}$ as the maximum storage capacity of the battery, we map duty cycles to different values of $\mathrm{EA}_{\mathrm{t}}$ as shown in table II.

It is to be noted that the discharge characteristics of the chargeable battery is non-linear. The value of discharge current affects the rate of battery discharge. This is known as the rate capacity effect. The rate capacity effect is defined using battery discharge parameter $\alpha$ that is current-dependent. 


\section{Sustaining the Life of Wireless Sensor Node with Energy Harvesting}

$\alpha$ is obtained by dividing the effective capacity of the battery at the particular discharge current by the maximum capacity of the battery. If $\mathrm{E}_{\mathrm{t}}$ is

TABLE II ENERGY HARVESTED AND DC VALUES

\begin{tabular}{|l|l|l|}
\hline ENERGY AVILABLE(J) & MODE & DC \\
\hline $\mathrm{E}_{\mathrm{A}}<0.1$ & Inactive & - \\
\hline $0.1 \leq \mathrm{E}_{\mathrm{A}}<1100$ & Active & 20 \\
\hline $1100 \leq \mathrm{E}_{\mathrm{A}}<1300$ & Active & 30 \\
\hline $1300 \leq \mathrm{E}_{\mathrm{A}}<1500$ & Active & 40 \\
\hline $1500 \leq \mathrm{E}_{\mathrm{A}}<1700$ & Active & 50 \\
\hline $1700 \leq \mathrm{E}_{\mathrm{A}}<1900$ & Active & 60 \\
\hline $1900 \leq \mathrm{E}_{\mathrm{A}}<2100$ & Active & 70 \\
\hline $2100 \leq \mathrm{E}_{\mathrm{A}}<2300$ & Active & 80 \\
\hline $2300 \leq \mathrm{E}_{\mathrm{A}}$ & Active & 90 \\
\hline
\end{tabular}

the energy remaining in a battery at time $t$ and $E_{t-1}$ that at previous time instant ( $\mathrm{t}-1)$ then $\mathrm{E}_{\mathrm{t}}$ can be defined as

$\mathrm{E}_{\mathrm{t}}=\mathrm{E}_{\mathrm{t}-1} /\left(1+\alpha \mathrm{I}_{\mathrm{d}}\right)$

where $I_{d}$ is the discharge current. $E_{t}$ and $E_{t-1}$ are expressed in Joules and $I_{d}$ in amperes.

\section{RESULTS AND DISCUSSION}

In order to understand the sustainability of the wireless node under solar energy harvesting scenario we have conducted simulation for a total duration of 96 hours and obtained values of $\mathrm{EA}_{\mathrm{t}}$ using (1) and (2) applying measured values of harvested energy. Both the scenarios EH1 and EH2 were considered for the study. Because of the diurnal nature of the source, the $\mathrm{EA}_{\mathrm{t}}$ values show a cyclic behavior. However, the node is able to sustain itself by operating at different duty cycle values in proportion to the amount of energy available. The maximum value of $\mathrm{EA}_{\mathrm{t}}$, in the case of scenario EH1 is limited by the storage capacity though, it could have been higher. This is reflected in the figure by the flat portion of the energy cycle for scenario EH1. This doesn't occur for the scenario $\mathrm{EH} 2$ because the value of energy harvested in this case is comparably less. From the cyclic nature of the remaining energy values in fig. 5, it can be seen that, with careful selection of duty cycle values in alignment with the energy available, the node is able to sustain its life. This, in turn, depends on the energy harvested.

We have also done a comparison of the performance with and without the limit on the battery storage capacity. The energy

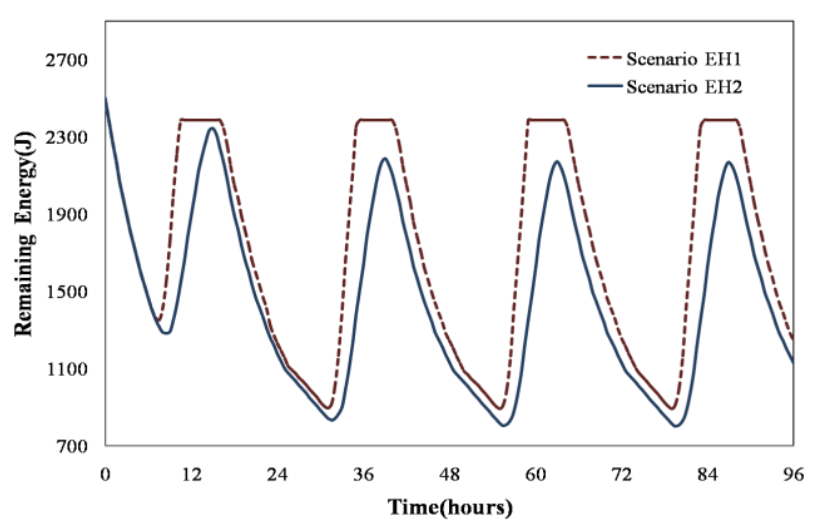

Fig. 5. Remaining energy over time

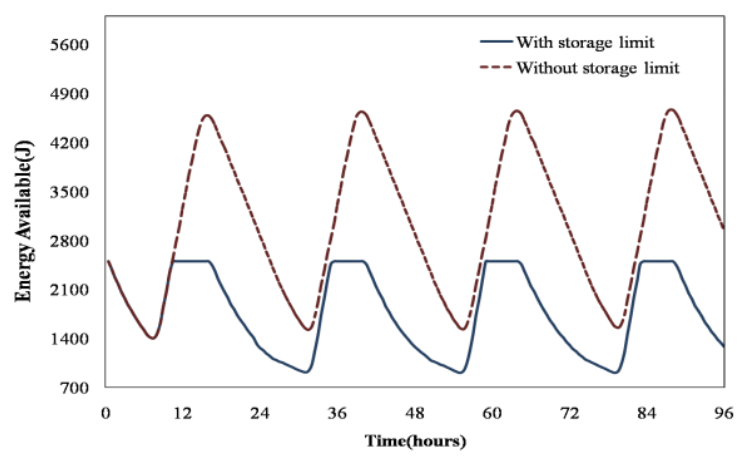

Fig. 6. Energy Cycle over a period of 96 hours: same activation time with and without limited storage

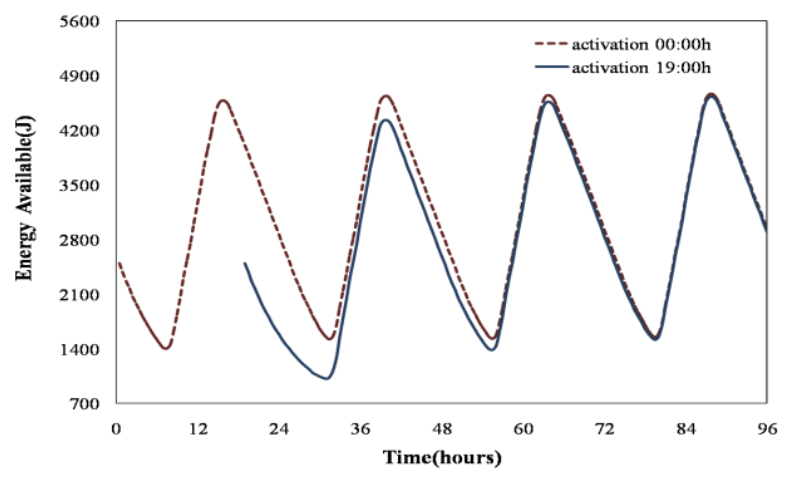

Fig. 7. Energy Cycle over a period of 96 hours: different activation time with maximum storage

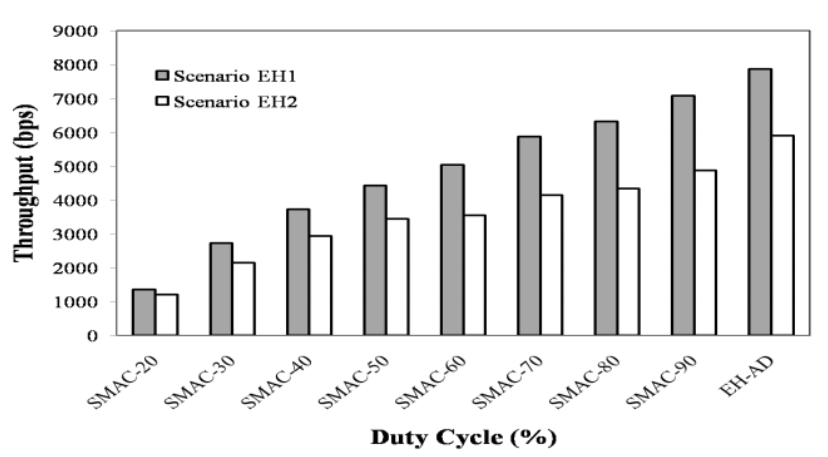

Fig. 8. Comparison of throughput with fixed cycle operations 
available is higher without the limit when the intensity of sunlight available is high. This is illustrated in figure 6 . Alternatively, we can increase the storage capacity to a higher value that utilizes the harvested energy to the maximum extent. This could improve the throughput further. However, the increase in the storage limit does not affect the performance under conditions where energy harvested is low.

Further studies were conducted to find out the effect of deployment time under the condition that there is no limit on storage capacity. Similar to the observations made in the case of fig. 6 , the energy stored in the battery will be discharged on a continuous basis without any harvesting if the node is activated during night period due to non availability of solar radiation. On the other hand, the discharge occurs along with energy harvesting when the node is activated during daytime. From fig. 7, it can be seen that the time of activation does not affect the life of the wireless node. After the initial time period, when the harvesting takes place both the energy graphs converge and the cycles continue without any difference. In comparison to the case with limited storage the initial time period required by the two graphs to get aligned is longer since the difference in energy available at the initial stage is higher.

We have found out the throughput of the network, achieved with the nodes operated at each of the duty cycle values and compared with the throughput obtained by varying the duty cycles in proportion to the energy harvested under the two different scenarios EH1 and EH2. This is illustrated in fig. 8. $\mathrm{EH}-\mathrm{AD}$ represents the proposed duty cycle adaptive approach discussed above. It can be seen that, under scenario EH1, there is a significant improvement in throughput varying from 1.86 times to 9.74 times that obtained from $90 \%$ to $20 \%$ duty cycles respectively. Similarly, the throughput is enhanced by a factor of 2.06 to 8.26 for scenario $\mathrm{EH} 2$

\section{CONCLUSION}

In this paper we have proposed an approach to sustain a wireless node using harvested solar energy. SMAC protocol commonly used in many WSN implementations was used to demonstrate the outcome. The findings were ascertained based on solar power measurements done at different weather conditions using small footprint solar panel suitable for a wireless node. We have shown that, in addition to extending the life of the node, the throughput of the energy harvesting node is considerably increased.

The effectiveness of the approach depends on understanding the behaviour of different components of the system such as characteristics of the energy source and energy consumption in the node, in addition to the specific MAC protocol. Based on the approach proposed, an optimisation algorithm can be developed for energy consumption and the study can be further extended to other MAC protocols and energy harvesting scenarios.

\section{REFERENCES}

[1] I. F. Akyildiz, W. Su, Y. Sankarasubramaniam, E. Cayirci, "A survey on sensor networks," IEEE Communications Magazine, Vol. 40, No. 8 , pp. 102-114, August 2002.
[2] David Culler, Deborah Estrin, Mani Srivastava, "Overview of Sensor Networks," Computer, August 2004.

[3] I. Demirkol, C. Ersoy, and F. Alagoz, "Energy efficient medium access control protocols for wireless sensor networks and its state-of-art," IEEE International Symposium on Industrial Electronics, Vol. 1, pp. 669-674, May 2004.

[4] Octarina Nur Samijayani, Hamzah Firdaus, Anwar Mujadin, "Solar Energy Harvesting for Wireless Sensor Networks Node," IEEE International Symposium on Electronics and Smart Devices (ISESD), 17-19 Oct. 2017, Yogyakarta, Indonesia.

[5] Greg Jackson; Simona Ciocoiu; Julie A. McCann, "Solar Energy Harvesting Optimization for Wireless Sensor Networks," 2017 IEEE Global Communications Conference, 4-8 Dec. 2017 Singapore.

[6] Amzar Omairi, Zool H. Ismail, Kumeresan A. Danapalasingam, Mohd Ibrahim, "Power Harvesting in Wireless Sensor Networks and its adaptation with Maximum Power Point Tracking: Current Technology and Future Directions," IEEE Internet of Things Journal Year: 2017, Volume: 4, Issue: 6

[7] Ou Yang, Wendi B Heinzelmzn, "Modeling and performance analysis for duty-cycled MAC protocols with applications to SMAC and XMAC," IEEE Transactions in Mobile Computing, vol. 11, No. 6, June 2012.

[8] Navid Tadayon, Sasan Khoshroo, Elaheh Askari, Honggang Wang, Howard Michel, "Power Management in SMAC-based Energy Harvesting Wireless Sensor Networks using Queuing Analysis," Journal of Network and Computer Applications 36(3), 1008-1017, 2013.

[9] Wei Ye, John Heidemann, Deborah Estrin, “An Energy-Efficient MAC Protocol for Wireless Sensor Networks", Proceedings of the 21st International Annual Joint Conference of the IEEE Computer and Communications Societies (INFOCOM 2002), New York, NY, USA, June, 2002.

\section{AUTHORS PROFILE}

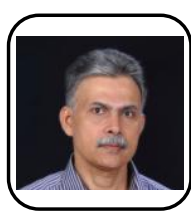

S. Viswanatha Rao is currently professor in the department of Electronics and Communication at Mar Baselios College of Engineering and Technology, Thiruvananthapuram. He completed his Bachelors and Masters in Engineering from Indian Institute of Science, Bangalore in 1985 and 1987 respectively and Bachelor of Science from Kerala University in 1983. He worked for the R\&D Division of the Indian Telephone Industries, Bangalore for 8 years from 1987 followed by 10 years in Institute for Infocomm Research $\left(I^{2} R\right)$, under the Agency for Science Technology and Research (ASTAR), Singapore at various levels from Senior Research Engineer to Programme Director. He has represented Singapore in the standardisation process of the International Standards Organisation(ISO) and EPC Global. His areas of interest include Wireless Communications, Wireless Sensor Networks and Digital Systems. He is a Senior Member of IEEE and Fellow of IETE

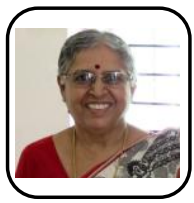

Dr Sakuntala S. Pillai received B. Tech Degree in Telecommunication Engineering, M. Tech degree in Microwave Engineering from College of Engineering, Trivandrum and $\mathrm{Ph}$. D Degree from University of Kerala. She was the Head of the Department of Electronics \& Communication, College of Engineering, Trivandrum from 1996-1998 and later worked as Director, LBS centre for Science and Technology, Trivandrum. She joined Mar Baselios College of Engineering \& Technology, Trivandrum as Head the Department of Electronics \& Communication in 2003. She worked there as Dean (R\&D) from 2008-2016. Her research interests include OFDMA, MIMO wireless systems, Error Correction Coding, Speech Coding etc. She is a Senior Member of IEEE, Fellow of IETE and Fellow of Institution of Engineers (India) and Life Member of Computer Society of India. 


\section{Sustaining the Life of Wireless Sensor Node with Energy Harvesting}

Dr Shiny G. received B. Tech Degree in Electronics and Communication Engineering from Madurai Kamaraj University in 1990 and M. Tech and Ph. D from University of Kerala in 1994 and 2013 respectively. Since 1998, she has been with the Department of Technical Education, Govt. of Kerala. Currently, she is a Professor in the Department of Electronics and Communication Engineering, College of Engineering Trivandrum. Her research interests include power electronics and drives, biomedical engineering and wireless communication systems. 